\title{
Diagnostic Imaging of Diffuse Infiltrative Disease of the Lung
}

\author{
Maurizio Zompatori a Claudio Bnà a Venerino Polettic Enrica Spaggiaria \\ Francesca Ormittia Elisa Calabrò ${ }^{b}$ Giuseppe Togninia Nicola Sverzellatia $^{a}$ \\ Dipartimento di Scienze Cliniche, aSezione di Diagnostica per Immagini e U.O. di Scienze Radiologiche, e \\ bSezione Clinica Pneumologica, Università degli Studi di Parma, Parma, e 'Dipartimento di Malattie dell'Apparato \\ Respiratorio e del Torace, Ospedale G.B. Morgagni, Forlì, Italy
}

\section{Key Words}

Chest X-ray · Diffuse infiltrative lung disease .

High-resolution CT · Imaging

\begin{abstract}
Plain chest radiography remains the first diagnostic approach to diffuse infiltrative lung disease but has limited diagnostic sensitivity and specificity. Many diseases remain occult or are not correctly assessed using chest Xray, appearing as a nonspecific 'reticulonodular pattern'. High-resolution CT (HRCT) is actually the recommended imaging technique in the diagnosis, assessment, and follow-up of these diseases, allowing also the evaluation of the effectiveness of the medical therapy and the selection of the type and the location of the biopsy when required. Appropriate techniques must be used to acquire highquality HRCT scans, with the thin collimation and high spatial reconstruction algorithm being the most important factors. A nodular pattern, linear and reticular opacities, cystic lesions, ground-glass opacities and consolidations are the most common HRCT patterns of diffuse infiltrative lung disease. This article reviews the role of chest radiography and HRCT in the diagnosis and assessment of these diseases, the technical aspects of HRCT, its clinical indications and the radiological pattern of the most common types of chronic diffuse infiltrative lung disease.
\end{abstract}

Copyright $@ 2004$ S. Karger AG, Basel

\section{Introduction}

The radiological diagnostic approach to diffuse infiltrative lung disease is classically based on chest X-ray evaluation. Among the most difficult tasks in radiology, chest X-rays remain unsurpassed in relation to its costs, radiation dose, availability, and ease of performance. However, chest radiography has substantial limitations: it is normal in 10-15 percent of symptomatic patients with proven infiltrative lung disease [1,2]; it can mimic interstitial disease in patients with different lung lesions (e.g. paraseptal emphysema and small-airway disease), and it is often quite nonspecific although abnormal.

Peripheral reticular, cystic, linear, nodular lesions and ground-glass density are the main radiological patterns used in the plain film interpretation of diffuse lung disease.Unfortunately, interstitial lung disease often appears in a non-specific 'reticulonodular' pattern [3]. The distribution of the opacities and the clinical history play a fundamental role in the assessment of nonspecific patterns.

In several studies, overall sensitivity and specificity of chest X-rays were 80 and $82 \%$, respectively, for the detection of diffuse lung disease; a confident diagnosis was possible in only $23 \%$ of cases, which proved to be correct in $77 \%[4]$.

Since 1982, when Todo et al. [5] first described the HRCT technique, the high correlation between HRCT

\footnotetext{
Prof. Maurizio Zompatori

Istituto di Scienze Radiologiche, Ospedale 'Rasori'

Via Rasori 10

IT-43100 Parma (Italy)

Tel. +390521 703432, Fax +390521 986352, E-Mail maurizio.zompatori@unipr.it
} 
findings and abnormalities seen on corresponding inflation-fixed lung specimens aroused great interest $[5,6]$. For the first time, the secondary pulmonary lobule and anatomic details as interlobar fissures, interlobular septa, centrilobular arterial branches and, in some cases, the thin intralobular septa could be correctly identified with a radiological method. Presently, more than 20 years after its introduction, HRCT is considered the best imaging tool in this field of application for the evaluation of the pulmonary interstitium and secondary pulmonary lobule to diagnose and assess diffuse lung disease.

Several authors have reported a sensitivity of $95 \%$ and a specificity approaching $100 \%$ for HRCT in disease detection $[4,7]$. A confident specific diagnosis has been possible in about one half of the cases, and these diagnoses proved correct in $93 \%$ compared to $77 \%$ with chest $\mathrm{X}$ rays.

Grenier et al. [8] assessed, using Bayesian analysis, the sequential value of clinical chest-radiographic and CT findings in classifying chronic diffuse infiltrative lung disease. The authors showed an incremental diagnostic benefit for HRCT: in a series of 100 patients with diffuse lung disease, an accurate diagnosis was made on the basis of clinical data in only $27 \%$ of the cases, increasing to $53 \%$ combined with radiographic findings and to $61 \%$ with CT data.

This article reviews the role of chest radiography and HRCT in the diagnosis and management of diffuse infiltrative lung diseases, the technical aspects of HRCT, its clinical indications and the radiological patterns of the most common diseases.

\section{Anatomic Considerations and Technical Approach}

In the high-contrast environment of the lung, the resolution of HRCT is $0.2-0.3 \mathrm{~mm}$. This 'high' resolution enables the radiological evaluation of anatomic and pathologic findings, similarly to the corresponding inflation-fixed lung specimens [9].

The smallest anatomical unit of the lung visible on HRCT is the secondary pulmonary lobule (Miller's lobule), i.e. the smallest area of lung parenchyma surrounded by connective tissue septa $[10,11]$ (fig. 1). These septa, known as interlobular septa, are best developed and visible in the periphery of the anterior, lateral, juxtamediastinal regions of the upper and middle lobes, and in the periphery of the anterior and diaphragmatic regions of the lower lobes. They are only occasionally visible when nor-

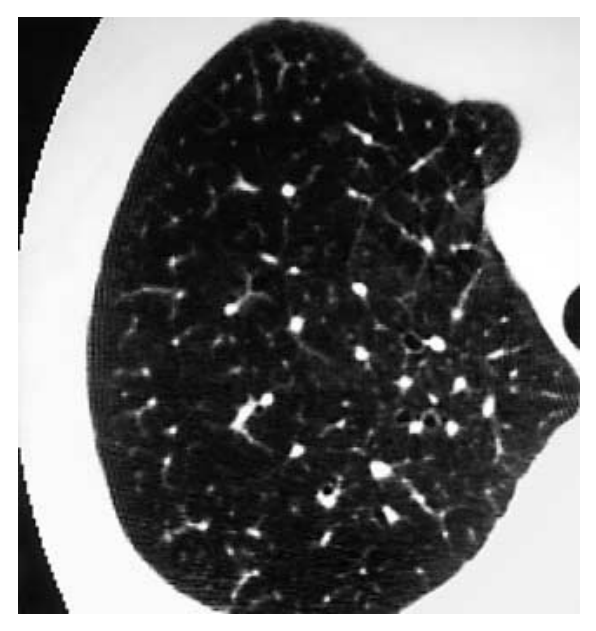

Fig. 1. HRCT feature of a secondary pulmonary lobule, the smallest area of lung parenchyma surrounded by connective tissue septa; in the middle of the lobule the centrilobular artery is correctly identified.

mal because, being $0.1 \mathrm{~mm}$ thick, they are at the lower limit of resolution of the technique [12].

Abnormal thickening of the interlobular septa, corresponding to the Kerley lines on chest radiograph, is always identified on HRCT. Similarly, abnormal thickening of the thin intralobular septa, of the axial interstitium surrounding the bronchovascular bundles and of the subpleural peripheral interstitium is always detectable. The centrilobular artery ( $1 \mathrm{~mm}$ thick in diameter) and the intralobular acinar arteries $(0.5 \mathrm{~mm}$ thick in diameter $)$ are correctly identified, whereas normal bronchioles supplying a secondary lobule ( $1 \mathrm{~mm}$ in diameter), having a wall thickness of approximately $0.15 \mathrm{~mm}$, are beyond the resolution of HRCT [13]. Normal airways in the secondary pulmonary lobules are not detected using HRCT.

\section{Clinical Role of HRCT in Diffuse Infiltrative Lung Disease}

HRCT plays a crucial role in the investigation of diffuse lung disease and is routinely used in the management of specific problems as [14]: (1) the detection and the assessment of the pattern, distribution and, in some cases, the activity and potential reversibility of diffuse lung disease, including those in symptomatic patients with normal or nonspecific chest X-ray; (2) the prediction of the response to therapy and the likelihood of survival; (3) the selection of the type and the location of the biopsy when required; (4) the follow-up of diseases and the evaluation 
Table 1. HRCT standard protocol for diffuse lung disease

Collimation: $1-1.5 \mathrm{~mm}$

Interval scan: $10 \mathrm{~mm}$

Scan time: $1-2 \mathrm{~s}$

High spatial frequency algorithm (bone algorithm)

Matrix $=512 \times 512$ pixels

Kilovolt peak $=120-140 \mathrm{kV}$

Milliamperage not $<200 \mathrm{~mA}$

Field of view as small as possible including both lungs

Window level $=-600 \mathrm{HU}$ (range -500 to $-900 \mathrm{HU}$ )

Width level 1,500 HU (range 1,100-2,000 HU)

of the effectiveness of medical therapy, and (5) the evaluation and the assessment of associated diseases such as emphysema, bronchiectasis, and bronchiolitis.

\section{Technical Features}

Appropriate techniques must be used to acquire highquality HRCT scans $[6,11,15-17]$. The goal of HRCT is to enhance the spatial resolution, in order to detect small structures and subtle abnormalities; the high contrast environment of the lung makes up for the loss of contrast resolution. The use of thin collimation and high spatial frequency reconstruction algorithm are the two most important factors that affect the spatial resolution and the quality of HRCT. Other relatively less important, controllable factors are field of view, kilovolt peak $(\mathrm{kVp}), \mathrm{mA}$, window level and width. Last but not least, the compliance and the ability of the technologist to coach the patient in breathing before and during the scan are fundamental to obtain high-quality and motion-free scans. An example of an HRCT standard protocol for diffuse lung disease is given in table 1. Most HRCT scans are obtained at full inspiration, with the patients in the supine position; only in selected cases prone and post-expiratory scans are required.

\section{Collimation}

Thin collimation and high spatial frequency algorithms are used to reduce volume averaging and to enhance the spatial resolution of HRCT images [18, 19]. The thinnest possible collimation (usually $1-1.5 \mathrm{~mm}$ ) should be used to optimize image resolution [11]. Thinner collimation $(<1 \mathrm{~mm})$ does not improve spatial resolution and reduces the signal-to-noise ratio. Thicker collimation $(>1.5-2 \mathrm{~mm})$ does not detect subtle density differences in lung parenchyma and compromises the correct evaluation of small nodules, ground-glass opacity (GGO), bronchiectasis, cyst walls and thickened interlobular septa. Although 2- to 3-mm-thick slices are still regarded as high resolution, subjective degradation in image quality is appreciable compared with thinner sections [20].

\section{Reconstruction Algorithm}

The reconstruction algorithm provides the recording frequency of the acquired scan data when the image data are created [18]. The standard or soft tissue reconstruction algorithms, used in conventional CT, are characterized by a low spatial frequency; these algorithms smooth the image, reduce the image noise and improve contrast resolution with a loss of spatial resolution. HRCT uses a high spatial frequency reconstruction algorithm (sharp or bone) to optimize anatomical details; the structure margins and tissue interfaces appear sharper and, although the noise is increased and contrast resolution is decreased, this is balanced by the high-contrast environment of the lung [11].

\section{Sampling}

HRCT is a sampling technique, in which the scans are performed at staggered intervals. In 'diffuse' infiltrative lung diseases, the pathological findings will be present for analysis on at least some of the images obtained by random sampling. However, it must be stressed that this 'sampling' technique is inappropriate for the detection of very small multifocal nodules such as metastases, single pulmonary nodules or focal disease. We perform standard HRCT scanning at $10-\mathrm{mm}$ intervals throughout the lungs, which is the most common scanning protocol. Decreasing the image spacing to obtain more images does not increase the diagnostic accuracy but prolongs the procedure and increases radiation doses. In selected protocols, imaging is performed at set anatomic levels such as the aortic arch, the carina or just above the diaphragm; if specialized studies (prone or expiratory HRCT scans) are necessary in addition to a routine supine study at full inspiration, the scan intervals are increased to $20 \mathrm{~mm}$ or only a few images are performed in the pathological areas, to decrease the additional radiation exposure.

\section{Patient Position and Respiration}

Generally, HRCT scans are obtained with the patient in the supine position at full inspiration, whereas the prone position is recommended in patients with normal or near-normal chest radiograph. They can eliminate the dependent atelectasis appearing as increased opacification or subtle abnormalities (linear densities, ill-defined 
areas of increased density or ground-glass attenuation) in the posterobasal segments of the lung, which may simulate early signs of an infiltrative disease [21]. Prone scans are not required if the chest radiograph is clearly abnormal (for example, in advanced interstitial fibrosis). Expiratory scans should be performed to differentiate the inhomogeneous attenuation (ground glass) of the lung parenchyma due to infiltrative lung disease from the mosaic perfusion due to air trapping or vascular obstruction [22-24].

\section{Target Reconstruction}

The smallest possible field of view that encompasses both lungs and the largest available matrix in the reconstruction of scan data (usually $512 \times 512$ ) should be used to decrease pixel size [15]. Decreasing pixel size relative to the area under evaluation means improving and maximizing spatial resolution in HRCT scanning. Magnifying the image does not further improve spatial resolution since only the pixel size is enlarged but not resolution.

\section{Window Width and Level}

There is not a single ideal window width and level combination for the evaluation of the lung parenchyma [15]; the appropriate window level, in case of suspected diffuse infiltrative disease, is between -500 and -900 Hounsfield units (HU), and the width level is between 1,100 and 2,000 HU. Traditional window widths (350$450 \mathrm{HU})$ and window levels (50 HU) should be used to evaluate the chest wall and mediastinum. Different window settings can be chosen in particular cases.

\section{Kilovolt Peak, Milliamperage and Scan Time}

Image noise is inversely proportional to the square root of the number of photons supplied during scanning. Noise can be decreased by changing $\mathrm{kVp}, \mathrm{mA}$ and scan time. Scan time should be short (usually $1 \mathrm{~s}$ ) to reduce movement artifacts as much as possible. Increasing the tube current $(\mathrm{mA})$ or $\mathrm{kVp}$ can help to reduce the image noise. In general, values of $\mathrm{mA}$ (varying from 200 to $300-$ for larger patients) and values of $\mathrm{kVp}$ (from 120 to 140) increase the quality of the images.

There had been some debates concerning the use of a reduced tube current to obtain HRCT images with reduced dose technique, because, while the dose is linearly related to tube current, any reduction in amperage increases the image noise and may somewhat invalidate the study.

Some researchers have reported that a minimum of $160 \mathrm{~mA}$ is necessary to identify small and subtle abnor-

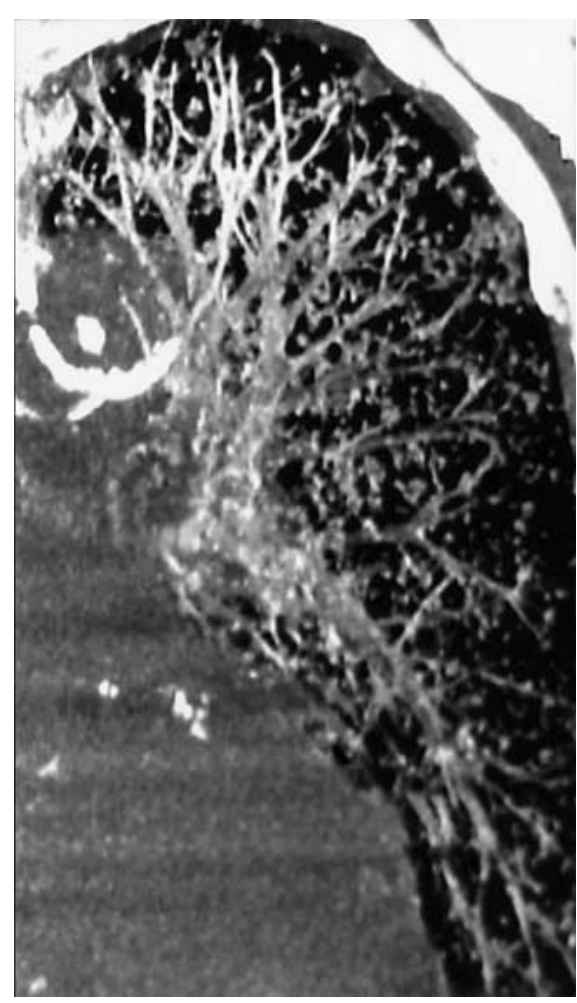

Fig. 2. A 56-year-old man with miliary tuberculosis. MIP image clearly shows the profusion and the distribution of micronodules and enables the distinction between micronodules and vessels.

malities (e.g. subpleural lines or GGO), whereas others found that the increased noise and the worse image quality of low-dose technique (as low as $40 \mathrm{~mA}$ ) do not preclude the detection of anatomical details [25-28]. The effective dose of a HRCT examination (1.5-mm sections, $10-\mathrm{mm}$ intervals, standard milliamperage and kilovoltage) is approximately 1 millisievert (mSv) versus $6.5 \mathrm{mSv}$ of the conventional (10-mm contiguous-section) $\mathrm{CT}$ chest scan.

\section{Volumetric HRCT}

Volumetric HRCT (VHRCT) is a new and promising technique to investigate diffuse pulmonary infiltrative disorders [29, 30]. VHRCT is carried out with the using spiral CT scanning of a stack of up to eight contiguous 1-mm sections. These are then reconstructed to produce a single image, using maximum (MIP) and minimum intensity projection (MINIP) reconstruction algorithms.

MIP images are actually considered superior to conventional HRCT in showing the profusion and the distribution of micronodules, and in enabling a better distinction between micronodules and vessels (fig. 2). 


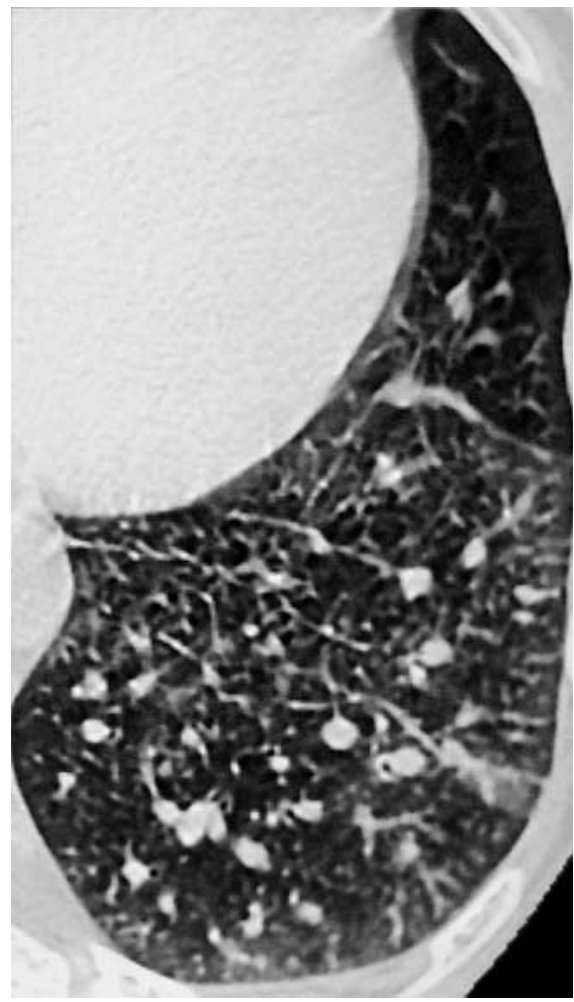

Fig. 3. Perilymphatic nodules in lymphangitic carcinomatosis. In a 60-year-old man with gastric cancer, HRCT shows nodular thickening of interlobular septa and nodules subpleural in location with a patchy distribution. The additional nodules in lung parenchyma represent hematogenous metastases.

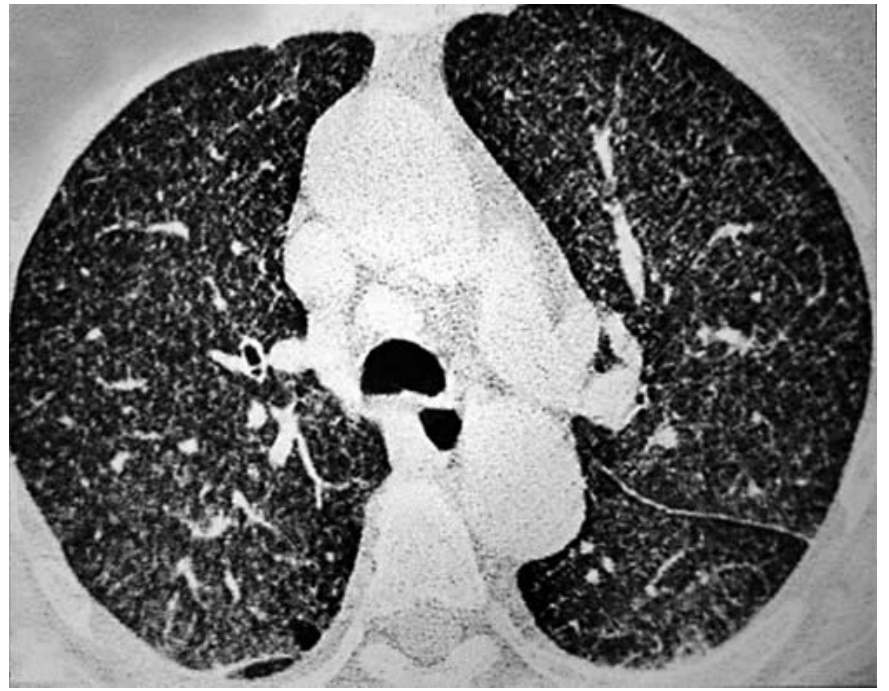

Fig. 4. 45-year-old man with miliary tuberculosis. Uniform and diffuse distribution of micronodules, expression of hematogenous dissemination of the disease.

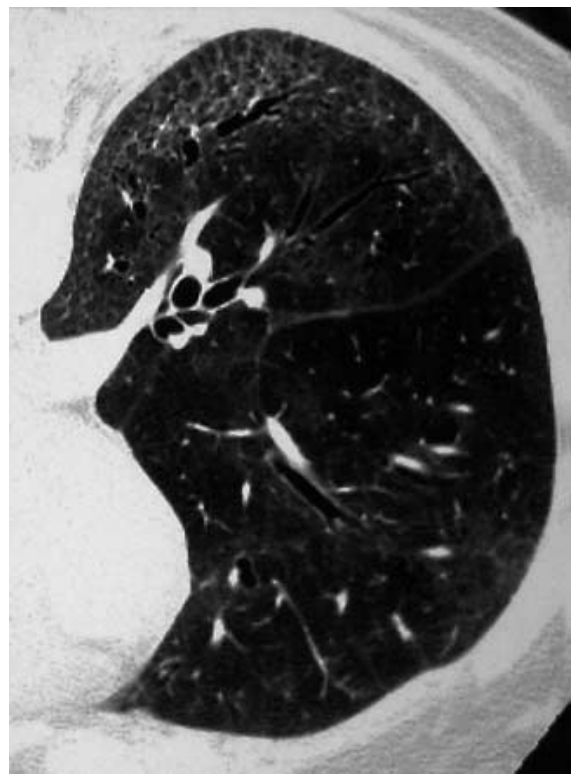

Fig. 5. 50-year-old man with UIP. HRCT scan performed with the patient in prone position shows irregular and intralobular septa with overlapping ground-glass density in the lower subpleural lung zones, with lobular architecture distortion, traction bronchiectasis and bronchiolectasis.

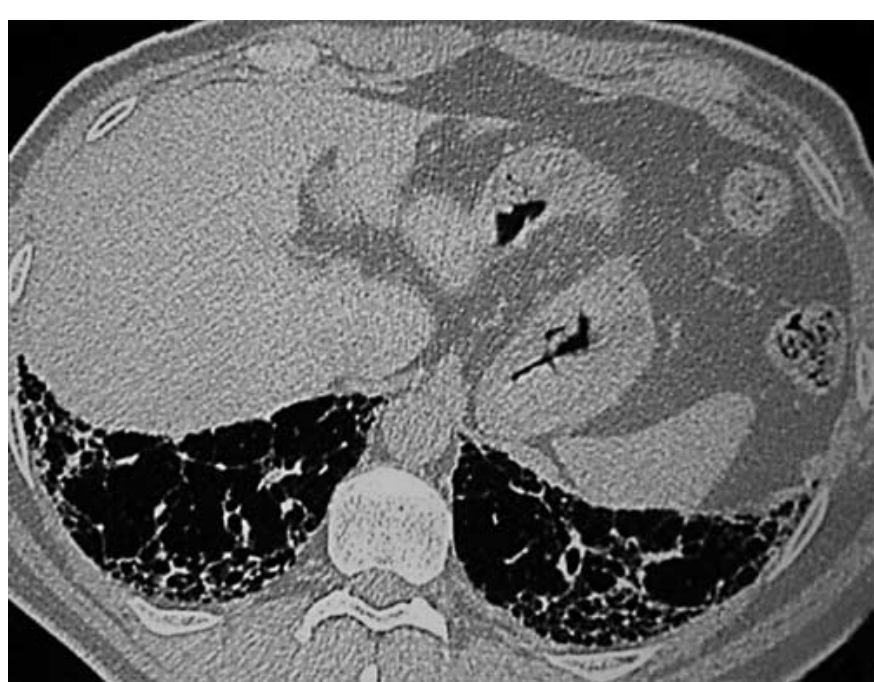

Fig. 6. HRCT scan demonstrates honeycombing in a 60 -year-old man with UIP in advanced stage. 
On a single 1-mm-thick section, a small, round subcentimetric structure may be interpreted as a lung nodule or as a vessel, whereas in a MIP reconstruction image the vessels can be recognized as linear and branching structures. MINIP can improve the detection of subtle areas of mosaic oligemia, emphysema or GGO and better differentiate the honeycomb pattern of pulmonary fibrosis from superinfected centrilobular emphysema.

\section{HRCT Patterns of Diffuse Infiltrative Lung Disease}

\section{Nodular Opacities}

Nodular patterns include airspace nodules and interstitial nodules, both referring to multiple round opacities varying in diameter from $1 \mathrm{~mm}$ to $1 \mathrm{~cm}$, with smooth or irregular margins in the presence or absence of cavitation [31]. According to their distribution, nodules can be classified as: perilymphatic nodules, centrilobular nodules and diffuse nodules. Perilymphatic nodules are most frequently seen in sarcoidosis, lymphangitic carcinomatosis and silicosis. These nodules are subpleural in location and are found along interlobular septa, interlobar fissures, and along bronchovascular bundles. Typically, they show a patchy distribution (fig. 3). Centrilobular nodules are found most frequently in extrinsic allergic alveolitis, respiratory bronchiolitis, Langerhans cell histiocytosis and bronchioloalveolar cell carcinoma. The distribution of these nodules is diffuse or patchy. Diffuse (random or perivascular) nodules have a uniform and diffuse distribution; these nodules indicate hematogenous dissemination and are typically seen in miliary tuberculosis, miliary fungal disease and hematogenous metastasis (fig. 4).

\section{Linear and Reticular Opacities}

Linear and reticular opacities, producing innumerable, interlacing line shadows suggesting a mesh are due to the thickening of the lung interstitium resulting from fibrosis, edema and infiltration of cells or other material $[10,31]$. The reticular pattern can be classified as large (thickening of interlobular septa), intermediate (concomitant with the honeycomb pattern) and fine (thickening of intralobular septa). Interlobular septal thickening can be smooth, nodular or irregular.

Smooth septal thickening is characteristic of pulmonary edema, but it can be also found in lymphangitic carcinomatosis. Less common causes include pulmonary hemorrhage, amyloidosis, and alveolar proteinosis. Nodular septal thickening is typical of lymphangitic carcino- matosis and less commonly seen in sarcoidosis and pneumoconiosis. Irregular septal thickening, demonstrating characteristic lobular architecture distortion, reflects interstitial fibrosis and is commonly present in usual interstitial pneumonitis (UIP) and asbestosis (fig. 5). Irregular linear opacities and a fine reticular pattern are the features of intralobular interstitium thickening. A reticular pattern can be seen in UIP, asbestosis and sarcoidosis.

\section{Cystic Lesions}

A cyst is defined as a thin-walled well-defined and circumscribed, air- or fluid-containing lesion, with an epithelial or fibrous wall $[10,32]$. The presence of a definable wall differentiates cysts from centrilobular emphysema. A cystic pattern can be indicative of: histiocytosis X, lymphangioleiomyomatosis and honeycombing. Honeycombing consists of a cystic air space having thick, clearly definable walls lined with bronchiolar epithelium, predominantly in the basal and subpleural areas [31]. It is a radiological feature of alveolar destruction and of the loss of the acinar architecture indicating the end stage of the lung (fig. 6). Honeycombing, a typical feature of pulmonary idiopathic fibrosis, allows a confident diagnosis of the disease.

\section{Ground-Glass Opacities}

GGO is defined as '... hazy increased attenuation of lung, with preservation of bronchial and vascular margins ...' [10] (fig. 7). It is a common but nonspecific finding indicating parenchymal abnormalities escaping the spatial resolution of HRCT. It can be due to partial filling of air spaces, interstitial thickening, partial collapse of alveoli, normal expiration and increased capillary blood volume. In the respective clinical and radiological context, it may lead to a specific diagnosis or more commonly represent areas of active inflammation following biopsy, but GGO can also be caused by microscopic fibrosis. Chronic infiltrative lung diseases typically associated with GGO include interstitial desquamative interstitial pneumonia (DIP), hypersensitivity pneumonitis and pulmonary alveolar proteinosis.

\section{Consolidation}

A consolidation (fig. 8) is characterized by a replacement of alveolar air by fluid, cells, tissue, or other substances that obscure underlying vascular structures [10]. It is frequently associated with air bronchograms. Consolidation is commonly seen in bronchiolitis obliterans with organizing pneumonia, chronic eosinophilic pneumonia, lipoid pneumonia, bronchoalveolar carcinoma and lymphoma. 


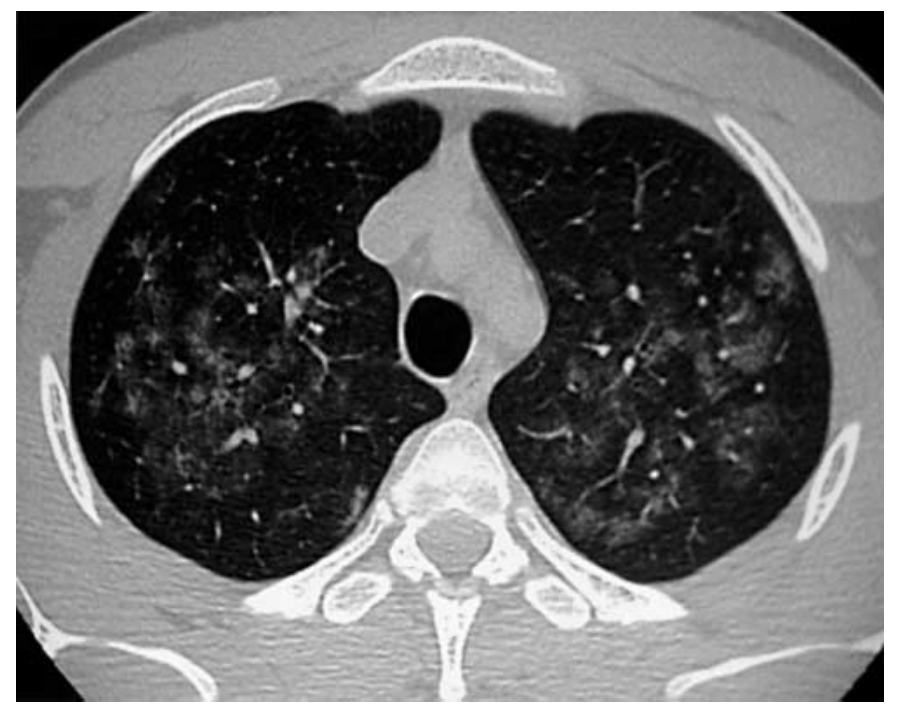

Fig. 7. HRCT scan shows GGO as hazy increased attenuation of the lung, with preservation of bronchial and vascular margins; the distribution of the lesions is patchy.

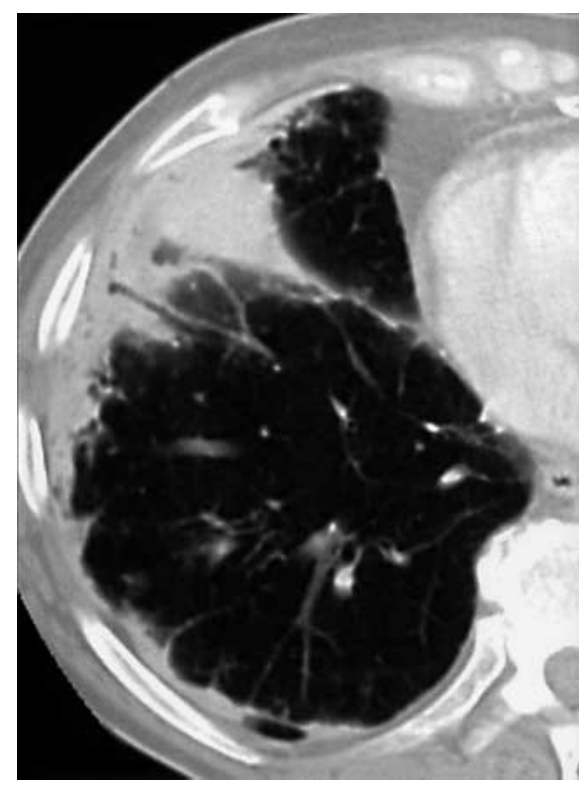

Fig. 8. 57-year-old man with bronchiolitis obliterans with organizing pneumonia. HRCT scan shows consolidations in the middle and inferior right lobe with air bronchogram.

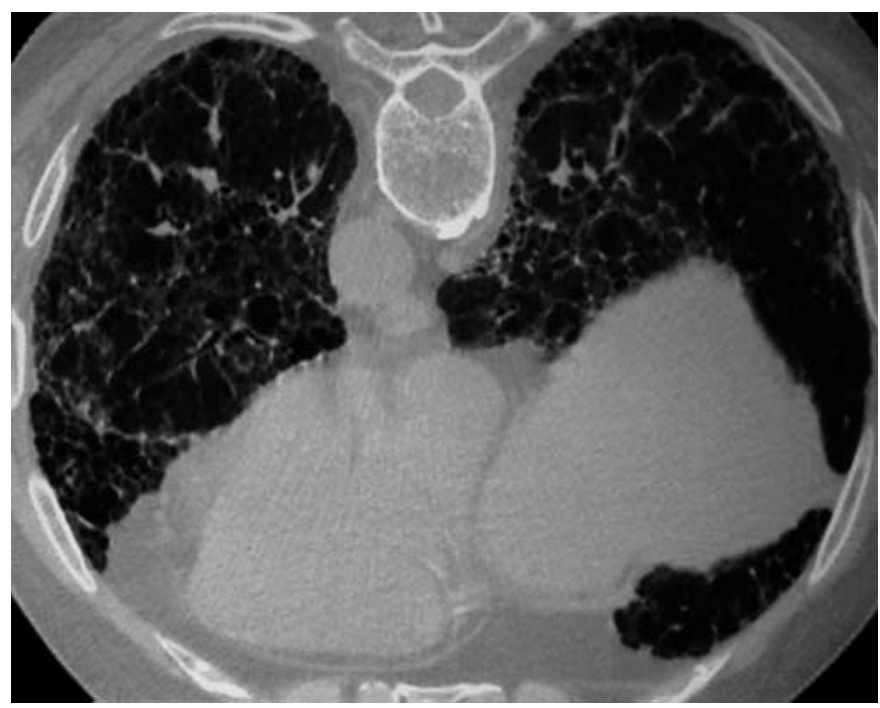

Fig. 9. 57-year-old man with UIP. HRCT scan in the prone position shows advanced disease with honeycombing, traction bronchiectasis, reticular pattern and GGO.

\section{Radiological Patterns of the Most Common Chronic Diffuse Infiltrative Lung Diseases}

\section{Idiopathic Interstitial Pneumonia}

UIP, DIP, acute interstitial pneumonia, nonspecific interstitial pneumonia and respiratory bronchiolitis with interstitial lung disease are a heterogeneous group of lesions with no well-defined etiology and thus classified as idiopathic interstitial pneumonia [33]. Diffuse inflammation and interstitial fibrosis showing a radiological reticular pattern, with lobular and intralobular architectural thickening and distortion, are the hallmarks of these diffuse infiltrative diseases. UIP is the most common type.

Chest radiographs show peripheral reticular pattern, characterized by interstitial opacities and small cystic spaces usually best seen in costophrenic angles and in the lower lung zones. In end-stage disease, the groups of lines forming a small cystic space may involve also the upper zones, even if a peripheral and basal predominance remains the key feature of the disease [3]. Fine or irregular intralobular linear opacities (reticular pattern), typically more severe and predominant in the lower lung zones with subpleural distribution, often associated with traction bronchiectasis and bronchiolectasis, are the typical HRCT features of UIP. Irregular pleural, vascular and bronchial interfaces are often seen.

The honeycombing pattern, characterized by the presence of cystic airspaces with thick (1-3 mm), clearly visi- 
ble fibrous walls, marks the final stage of the disease (end stage of the lung), allowing a confident diagnosis of lung fibrosis (fig. 9). The honeycombing pattern is present in 80-95\% of patients with UIP. The absence of this pattern should suggest an alternative diagnosis. Honeycombing, as the other lesions, are subpleural and predominantly distributed in the lower lobe.

Cystic spaces, ranging in size from several millimeters to several centimeters and often air filled, are layered in multiple rows and stacked along the pleural surface; if connected with the bronchioli, they can slowly increase in diameter, mimicking bullae of emphysema, and can also rupture resulting in pneumothorax.

The association of centrilobular and paraseptal emphysema with lung fibrosis, as found in heavy smokers, may help to detect clinical characteristics by HRCT. HRCT often depicts in the same patient and in the same area of the lung a patchy or geographic distribution of the lesions, with reticular pattern intermingled with normal lung parenchyma, fibrosis and GGO. The often associated ground-glass attenuation, usually less extensive in the reticular or cystic pattern, is common and can represent fibrosis with microscopic honeycombing or more active inflammation areas [34].

However, the significance of ground-glass areas in patients with UIP has been subject to discussion [35]. In $10 \%$ of the patients, ground-glass attenuation predominates or is the only lesion. Consequently, alternative diagnoses, which include idiopathic interstitial pneumonia (specially DIP or nonspecific interstitial pneumonia), hypersensitivity pneumonitis, edema, alveolar proteinosis and bronchioloalveolar cell carcinoma, should also be borne in mind [36]. In these patients, biopsy is required to confirm the diagnosis. The ground-glass areas, if predominant and not associated with fibrosis, may be completely or partially amenable to therapy, whereas they develop in a typical honeycombing pattern if untreated. Several studies [37-40] have suggested that in patients with UIP the ground-glass attenuation areas (usually concomitant with a reticular pattern) precede the development of lung fibrosis, which progresses despite therapy.

HRCT and functional tests are actually used to follow up UIP; the extent of parenchymal lesions and the degree of volume loss correlate with the severity of functional impairment. HRCT shows mediastinal lymph node enlargement in $70-90 \%$ of patients with UIP.

\section{Collagen Vascular Disorders}

Collagen vascular disorders as scleroderma or rheumatoid arthritis (AR) can develop into an interstitial lung disease showing a peripheral reticular pattern. The differential diagnosis with UIP can be difficult, but the known history of the disease, often preceding the development of interstitial lung lesions, and serologic data may suggest the correct diagnosis.

HRCT shows a better sensitivity and specificity than chest X-rays [41]. Schurawitzki et al. [42], in a series of 23 patients with scleroderma, reported a $91 \%$ sensitivity of HRCT in the detection of parenchymal involvement, compared with 39\% using chest X-rays. HRCT features of scleroderma include an irregular thickening of interlobular septa, subpleural lines well seen as linear and curvilinear opacities over $2 \mathrm{~cm}$ in length due to the thickening of many interlobular septa and intralobular interstitial thickening producing increased intralobular attenuation. HRCT can reveal additional signs of scleroderma as pleural thickening, mediastinal adenopathy, and esophageal dilatation.

The radiological and histological features of AR are not distinguishable from those of UIP. Interstitial lesions as basal linear opacities and the reticulonodular pattern, and honeycombing in the end stage of the disease are seen on chest X-rays in $2-10 \%$ of cases with AR, whereas the functional impairment is present in $30-40 \%$ of cases. HRCT reveals intralobular and interlobular interstitial thickenings, distortions of parenchymal structure, honeycomb lesions in the advanced disease and more significant signs of AR as bronchiectasis, bronchiolectasis, centrilobular micronodules and mosaic perfusion with expiratory air trapping.

\section{DIP and Respiratory Bronchiolitis with Interstitial \\ Lung Disease}

DIP has been recently associated with respiratory brochiolitis-associated disease (RBILD) as a single entity with identical lesions and HRCT features, differing only in the severity and extent of the lesions (RBILD = mild/ early DIP) [43]. DIP and RBILD represent actually a different degree of involvement of the small airways and parenchymal reaction to cigarette smoking, and are therefore strongly related. Usually, patients respond to therapy, and the disease can be reversible if the patients stop smoking.

Chest radiographs may look normal, but basal or diffuse slender opacities with fine reticular and micronodular patterns are more often (70\% of cases) found [44]; these findings are nonspecific and similar to the 'dirty lung' of the heavy smokers [45]. HRCT shows ground glass bilaterally, peripherally and predominantly in the lower lung zones, with a diffuse or patchy distribution as most typical features of DIP [41] (fig. 10). 


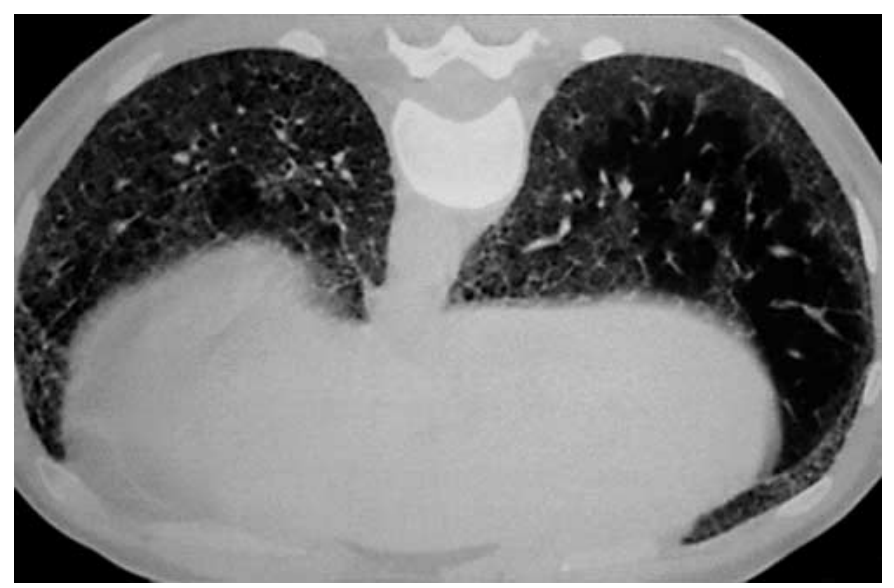

Fig. 10. 50-year-old patient with DIP. HRCT scan with the patient in the prone position shows bilateral diffuse GGO and reticular peripheral opacities in the lower lung zones.

Fibrosis (reticular peripheral pattern), subpleural and basal in location, is seen in $50 \%$ of the patients, even though it is mild and much more limited in extent compared with that of the UIP. Small cysts, probably due to bronchiolar obstruction, can bee seen in the ground-glass attenuation areas. These findings can simulate a very mild honeycombing and may respond to therapy, differently from UIP honeycombing.

\section{Asbestosis}

In patients with a history of asbestos exposure (generally at work), HRCT findings of inter- and intralobular small nodules and branching opacities, septal thickening, subpleural and parenchymal bands, pleural plaques, diffuse pleural thickening, and areas of mosaic oligemia are fairly specific features resulting in the diagnosis of asbestosis [46]. Asbestos-related plaques or fibrothorax, when present, can be used to confirm the diagnosis (fig. 11).

With respect to chest X-rays, HRCT can better reveal the early signs of asbestosis as peripheral centrilobular micronodules, thin and basal reticular patterns, subpleural lines (thin curvilinear opacities paralleling the pleural surface) and parenchymal bands.

In the literature, the percentage of asymptomatic patients with a history of asbestos exposure having normal chest X-rays but HRCT findings typical for asbestosis varies from 5 to $20-30 \%$ [47-49]. In advanced cases, the subpleural and basal distribution of fibrosis in asbestosis resembles the lung fibrosis of UIP, although asbestosis is

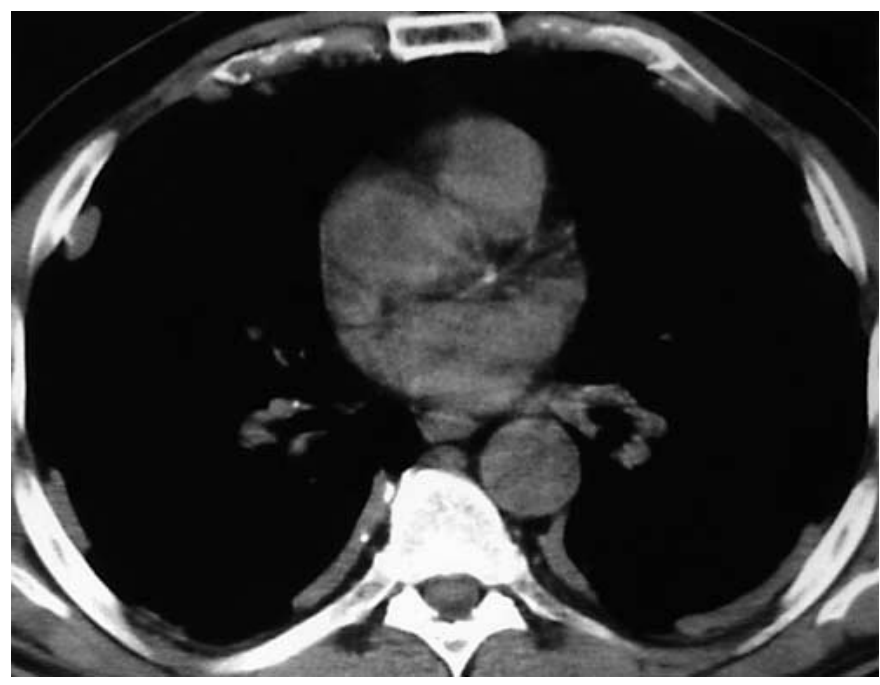

Fig. 11. 40-year-old man with asbestosis. The chest wall and mediastinum evaluation with mediastinal window widths and levels reveals numerous pleural plaques and pleural thickenings.

usually associated with pleural plaques and diffuse pleural thickenings. Pleural involvement is strongly correlated with the severity of fibrosis. Ground-glass attenuation areas are uncommon and limited. Even if none of the radiological signs is pathognomonic of asbestosis, HRCT is often performed to clarify the abnormal pattern seen on chest X-ray or when there is no correlation between functional impairment and radiological findings.

\section{Lymphangitic Carcinomatosis}

Chest-X ray findings of lymphatic carcinomatosis (LC) are nonspecific, and in many patients (23-50\%) with symptoms and proven disease, chest radiographs can be normal [50-54]. Kerley lines A and B, expression of peripheral interlobular septa thickening, subpleural effusion, a diffuse reticular pattern involving one or both lobes of the lung with or without adenopathy, and sometimes pleural and pericardial thickening and/or effusion, are the most common chest X-ray features of LC.

HRCT can correctly detect lymphangitic spread in up to $50 \%$ of patients with symptoms and normal chest $\mathrm{X}$ ray. It shows thickening of the interlobular septa, of the bronchovascular and subpleural interstitium, and thickening of bronchovascular core structures in the secondary lobule, without the fibrotic distortion of lung parenchyma (fig. 12).

The affected interstitial structures can be nodular, beaded or smooth, producing a typical reticulonodular pattern [17]; these findings can be bilateral and diffuse or 


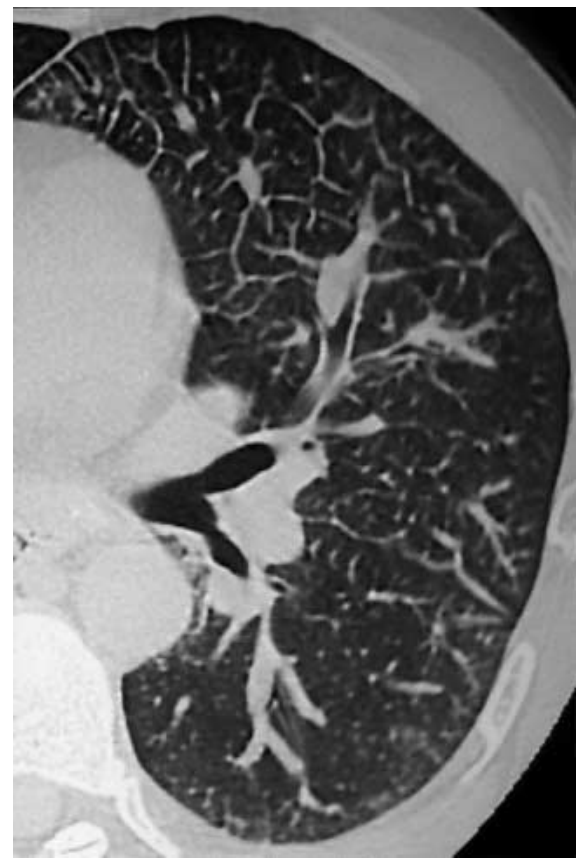

Fig. 12. Characteristic presentation of lymphangitic carcinomatosis in a 48-year-old woman with known breast cancer. HRCT scan shows a thickening of interlobular septa and bronchovascular bundles without fibrotic distortion of the lung parenchyma.

focal and unilateral as in the lymphangitic carcinomatosis due to a primary lung cancer. Associated findings are intrapulmonary nodules (hematogenous metastases), hilar or mediastinal lymphadenopathy and pleural effusion.

In patients with a clinical history of primary carcinoma of the breast, lung, stomach, pancreas, cervix, prostate or thyroid, or in patients with metastases of an adenocarcinoma of unknown primary site, HRCT features of LC are characteristic and a confident diagnosis can almost always be obtained.

The absence of distortion of lung parenchyma and the more linear, smooth septal involvement, despite the extensive involvement, differentiates LC from sarcoidosis, which may show similar findings.

\section{Histiocytosis $X$}

Histiocytosis X, otherwise know as Langerhans' cell histiocytosis, eosinophilic granuloma and Langerhans' cell granulomatosis of the lung, is a smoking-related lung disease with so far unknown etiology that can improve or resolve if the patients quit smoking. Systemic, skeletal and visceral manifestations are seen in $40 \%$ of the patients, but in the remainder $(60 \%)$ the lung is the only site

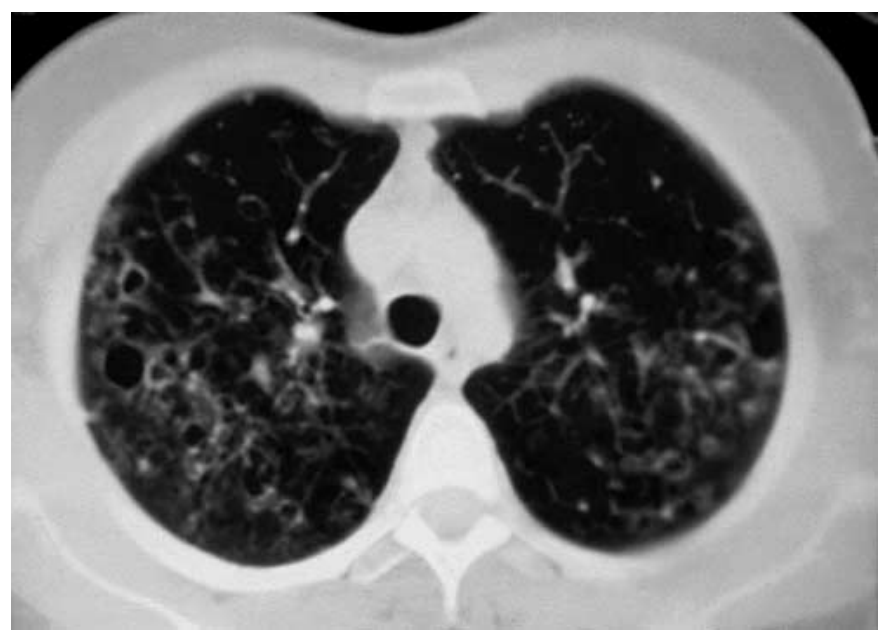

Fig. 13. 40-year-old man with histiocytosis X. HRCT scan shows round or bizarre-shaped cystic airspaces of different sizes. There are also nodules and fibrotic strands in the lung parenchyma.

of involvement, with a typical upper-lobe predominance. Chest X-rays shows nodular, cystic, reticular and reticulonodular lesions, more often symmetrically involving the middle and upper lung zones, and frequently sparing the costophrenic sulci.

HRCT is much more specific and sensitive than chest $\mathrm{X}$-rays in the evaluation of the extent and activity of the disease with a high degree of correlation with functional impairment; Grenier et al. [55] showed a correct first choice diagnosis in $90 \%$ using HRCT compared to $60 \%$ using chest X-ray.

The early stage of the disease is characterized by a bronchiolocentric granulomatous reaction with involvement of the bronchiolar wall and blood vessels. The corresponding HRCT appearance is a nodular pattern: peribronchiolar and centrilobular nodules usually $<5 \mathrm{~mm}$ in diameter (larger nodules $\geq 10 \mathrm{~mm}$ in diameter can occur but are uncommon). Depending on the disease activity, nodules can be few or numerous.

In progressive disease, cavitated (hollow) nodules, bronchiolar wall thickening and cystic airspaces with walls that range from barely perceptible to several millimeters thick occur [17] (fig. 13). During remission, only thin-walled cysts can be found. 


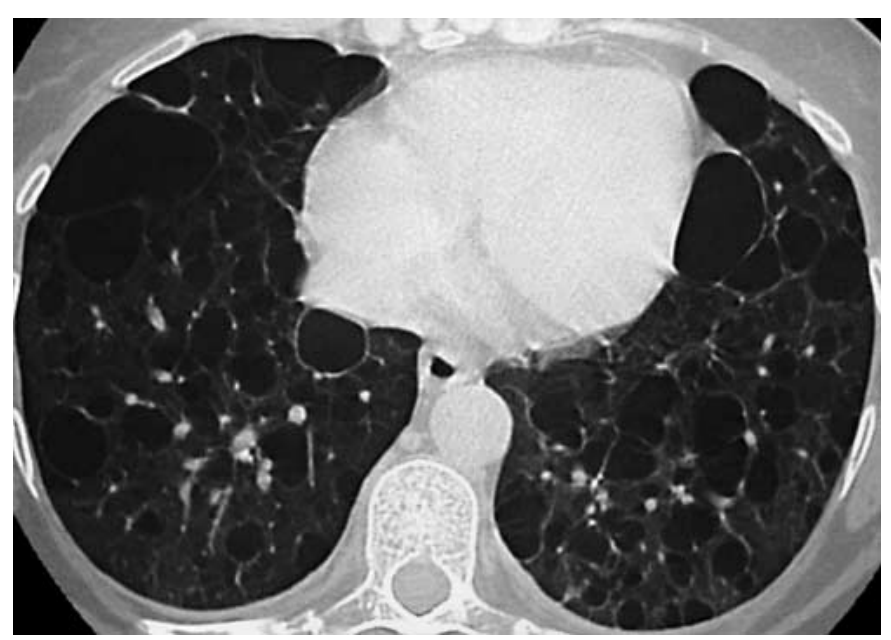

Fig. 14. Typical HRCT features of lymphangiomyomatosis in a 34year-old woman: innumerable thin-walled cysts completely replace the lung parenchyma.

Cysts, identified in $80 \%$ of the patients, are the more typical finding of the disease. They may be round, lobulated, irregular or bizarre in shape and are usually $<10 \mathrm{~mm}$ in diameter but may coalesce and then become $>20 \mathrm{~mm}$. Rupture of cysts in the pleural space is the cause of the frequent episodes of pneumothorax. Nodules and cysts can occur solely, but in the majority of patients they are concomitantly found. Occasionally, patchy or diffuse GGO may be seen. The disease shows upper-lobe predominance with sparing of the lung bases and the costophrenic sulci; the lung volume is normal or, sometimes, increased.

The HRCT findings of irregular, bizarre cysts and peribronchiolar, centrilobular nodules involving the upper lobes in a cigarette smoker permit a confident diagnosis of histiocytosis $\mathrm{X}$.

\section{Lymphangiomyomatosis and Tuberous Sclerosis}

Lymphangiomyomatosis (LAM) is a disease restricted to women of childbearing age, and it is not associated with smoking. A hamartomatous progressive proliferation of smooth muscle cells along the bronchovascular bundles, lymphatics and pulmonary veins is the histopathological finding of LAM; the same radiological and pathological findings are found in patients with tuberous sclerosis showing pulmonary involvement ( $1 \%$ of the cases).

Marked hyperexpansion of the lungs, spontaneous pneumothorax, chylothorax and hemoptysis are frequent clinical manifestations of the disease. In $70 \%$ of the cases, pleural and parenchymal abnormalities are clearly visible on chest X-ray: a diffuse/symmetric reticular pattern or one resembling honeycombing with increased or normal pulmonary volumes and expiratory air trapping is often found. Pleural effusions and recurrent pneumothoraces are frequent.

It is important to underline that the reticular and honeycombing patterns seen on chest X-ray are due to superimposed cystic lesions, which are numerous and nearly uniform in diameter; in LAM, the fibrotic component is absent or not relevant. HRCT is significantly superior to the chest radiograph in the evaluation of the extension and the distribution of cystic lesions; furthermore, HRCT findings correlate well with clinical and functional impairments.

HRCT features of LAM are typical and characteristic: innumerable thin-walled cysts ranging in diameter from $2 \mathrm{~mm}$ to $5 \mathrm{~cm}$, surrounded by mostly normal parenchyma [35, 41] (fig. 14). The cystic lesions are uniformly and symmetrically distributed in the upper and lower lobes, without sparing of lung parenchyma; vessels are displaced at the periphery of the cysts. The wall thickness of the cysts ranges from rarely perceptible to few millimeters. Typical is also the absence of signs of fibrosis, and only occasionally are nodules reported.

Patchy GGO (focal hemorrhage due to obstruction of small pulmonary veins) or the thickening of interlobular septa (interstitial edema due to obstruction of pulmonary lymphatics) can be seen. Other HRCT features include: mediastinal or hilar adenopathy, chylous pericardial, pleural and peritoneal effusions and spontaneous pneumothoraces due to the rupture of cysts in the pleural spaces.

In suspect patients (women of childbearing age with recurrent pneumothoraces, chylous effusions, obstructive or functional impairment), a HRCT study is strongly suggested and may be supplemented eventually, if tuberous sclerosis is suspected, with CT of the head and the abdomen. The presence of uniformly sized, thin-walled cysts distributed from the center to the periphery and from the apex to the lower lung zone is virtually pathognomonic for LAM.

\section{Sarcoidosis}

Sarcoidosis is a chronic, multisystemic disorder of unknown cause characterized - in affected organs - by an accumulation of $\mathrm{T}$ lymphocytes and mononuclear phagocytes, noncaseating epithelioid granulomas and derangement of the normal tissue architecture [56].

Pulmonary manifestations occur in $90 \%$ of the patients, and the classic presentation consists of enlarged 
hilar and mediastinal lymph nodes, with or without lung parenchymal involvement. In the affected lung parenchyma, inflammatory cells and sarcoid granulomas cluster, forming nodules that distort the wall of the alveoli, bronchi and blood vessels, altering the normal gas exchange in advanced cases. In the group of granulomatous diseases manifesting on chest X-ray with a nodular interstitial pattern, sarcoidosis is among the most common.

Frequently, the diagnosis is firstly found following chest radiography, and therefore staging of the disease is based on chest X-ray findings: stage I = hilar and mediastinal lymphadenopathy; stage II = mediastinal lymphadenopathy in association with nodular interstitial lung disease; stage III = nodular interstitial disease without lymphadenopathy, and stage IV = lung fibrosis.

Bilateral, symmetric, and right paratracheal lymphadenopathy, with or without parenchymal involvement, is seen in $60-70 \%$ of the patients. Parenchymal lesions are detected as numerous tiny nodular densities, varying from 1 to $5 \mathrm{~mm}$ in diameter, rarely larger, associated with linear and irregular opacities. In stage IV, upward retraction of the hilum combined with a transverse band of fibrosis originating in the hilum is commonly seen.

It is well known that sarcoidosis greatly varies in radiological appearance and that it can mimic many other diffuse infiltrative diseases. However, the typical HRCT features of active sarcoidosis [57] include several small, welldefined nodules showing 'perilymphatic' distribution with respect to their subpleural location (mimicking pleural plaques) along interlobular septa, interlobar fissures and along bronchovascular bundles (fig. 15a). In the lobular core, the nodules are adjacent to vessels which appear irregularly enlarged. Irregularly shaped nodular lesions predominate in the perihilar and dorsal region with relative sparing of the lung periphery, but they may also be found throughout both lungs in the upper and middle zones, or may be grouped uni- or bilaterally in small areas.

Large nodules (1-4 cm in diameter) and consolidations with air bronchogram (so-called 'alveolar sarcoid pattern') are found in about $15-25 \%$ of the patients [57-60] (fig. $15 \mathrm{~b}$ ). Roughly $20 \%$ of the patients develop a pulmonary fibrosis: conglomerated parahilar masses distorting bronchi and vessels, traction bronchiectasis, septal thickening and honeycombing [35].

HRCT frequently shows ground-glass attenuation areas with patchy distribution, often associated with nodular and fibrotic patterns, as an expression of interstitial inflammation and early parenchymal fibrosis. CT shows hilar and mediastinal adenopathy in $80 \%$ of the patients
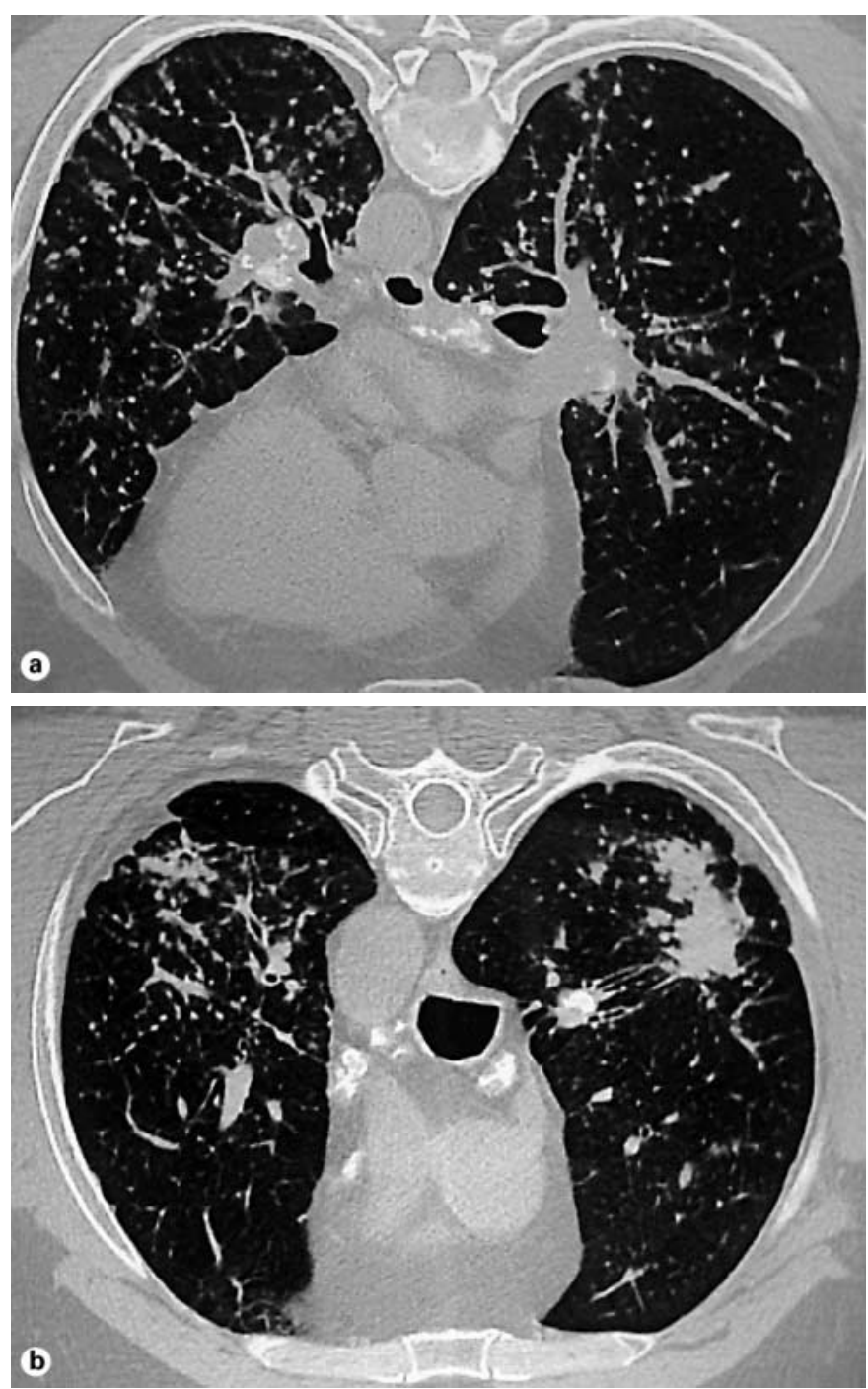

Fig. 15. HRCT of a 38-year-old man with active sarcoidosis in the prone position. a HRCT scan shows several small well-defined nodules with perilymphatic distribution and hilar and mediastinal lymph-nodal calcification. b HRCT shows large nodules and consolidations.

with normal chest X-ray and detects lymph-nodal calcifications in about $50 \%$ of old sarcoidosis. Several authors underlined that intrapulmonary microscopic granulomas cannot be detected by HRCT $[59,61,62]$.

However, CT is more sensitive and specific than chest radiography in detecting early parenchymal abnormalities even when radiography only indicates adenopathy $[17,41]$. For example, a loss of volume in the posterior zones of the upper lobes, well recognized with HRCT as a posterior displacement of the bronchi, is an important early sign of lung distortion. 


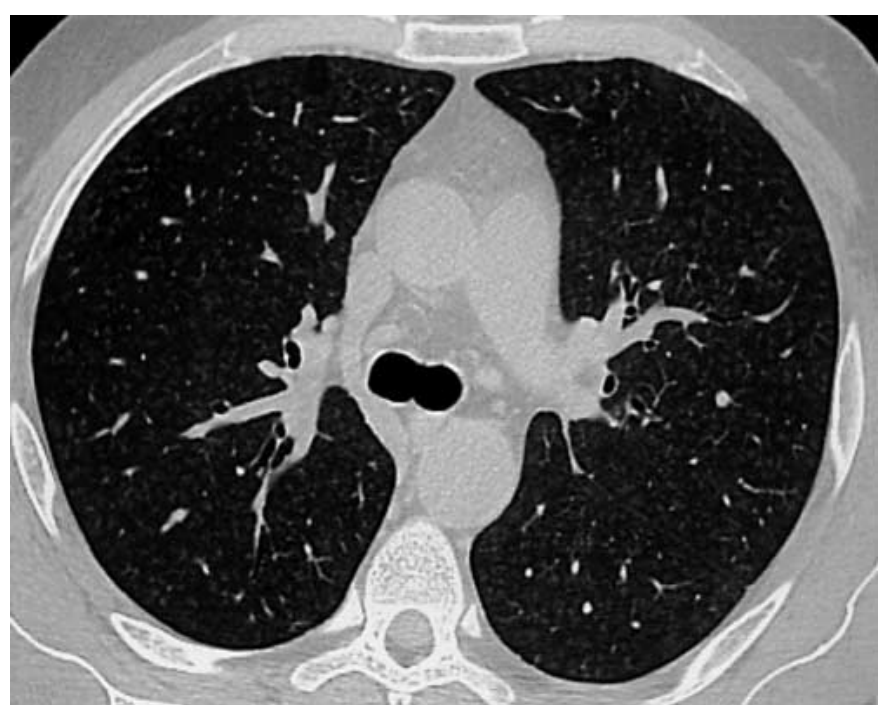

Fig. 16. 37-year-old patient with subacute allergic extrinsic alveolitis. HRCT scan shows small ill-defined centrilobular nodules.

The typical features of sarcoidosis are well recognized. Rarely, there are problems in the differential diagnosis with diseases like LC, silicosis and coal worker's pneumoconiosis, which have a similar nodular perilymphatic pattern. The predominant distribution of nodules along the perihilar bronchovascular bundles and in the subpleural zones, and the presence of air bronchograms in the fibrotic areas are the relevant features in the diagnostic process.

There are some debates concerning the superiority of HRCT compared to radiography in correlating (not strictly) the radiological findings with functional impairment [58, 63-65]. However, as Hansell et al. [66] have shown, the obstructive or mixed functional impairment, found in advanced disease, appears to correlate better with the presence and extension of fibrotic lesions than with the extent and distribution of nodules and consolidations.

\section{Silicosis and Coal Worker's Pneumoconiosis}

Silicosis and coal worker's pneumoconiosis, due to inhalation of dust-containing crystallized silicon dioxide and coal dust, respectively, have enormous social, economic, and medical significance in the industrial countries [67]. Radiological features of silicosis and coal worker's pneumoconiosis cannot be distinguished; chest X-ray shows a predominance of nodular opacities in the upper lung zones, $1-10 \mathrm{~mm}$ in diameter, typically very sharply marginated resembling sand.
In advanced disease, a region of fibrosis will develop in the upper lobes with cicatricial emphysema peripheral to the mass. Characteristic findings of HRCT are [41] small (2-5 mm), well-defined, typically centrilobular nodules scattered uniformly in the perihilar and dorsal lung zones, with upper lobe predominance.

The nodules may calcify with time and/or increase in diameter, clustering and coalescing in large nodules or large conglomerated masses with irregular margins, socalled silicomas. The process reflects the evolution from simple to complicated silicosis. These masses of pulmonary massive fibrosis are typically parahilar in location, egg shaped and associated with fibrotic distortion of parenchyma and paracicatricial emphysema.

Often, enlarged thoracic lymph nodes, with 'eggshell' calcifications, are noted. Even if the use of HRCT is not yet standardized, this technique has a better sensitivity and specificity than chest radiography in the detection of the earlier signs of pathology. So, it can rule out the parenchymal involvement or confirm the presence of the disease in those cases with normal or uncertain X-ray findings.

The extent of emphysema correlates (as in all the granulomatous diseases) better with functional impairment than the degree of nodular profusion.

\section{Allergic Extrinsic Alveolitis}

(Hypersensitivity Pneumonitis)

Hypersensitivity pneumonitis, or allergic extrinsic alveolitis, is an immunologically induced inflammation of the lung parenchyma involving alveolar walls and terminal airways following repeated inhalation of organic or other agents by a susceptible host [68]. Chest X-rays are neither specific nor distinctive. In the acute or subacute phase, findings vary from poorly defined, patchy, or diffuse infiltrates to discrete nodular infiltrates and, in the chronic phase, a reticulonodular infiltrate. The HRCT feature is proteiform, depending on the stage of the disease.

Diffuse, bilateral ground-glass attenuation areas with geographic distribution are the most common findings in the acute phase. Findings of mosaic perfusion and air trapping due to bronchiolar obstruction, better or only recognized on expiratory scans, can be frequently found, and sometimes they are the predominant features.

HRCT is particularly helpful in the subacute stage of allergic extrinsic alveolitis: GGO, small ill-defined centrilobular nodules (1-5 mm), which predominate in the lower zones, and slightly thickened bronchial walls are the typical findings [41] (fig. 16). Pulmonary fibrotic lesions 
characterize chronic allergic extrinsic alveolitis: irregular reticular opacities, intralobular interstitial and interlobular septal thickening, traction bronchiectasis and bronchiolectasis, and honeycombing are often seen. The lesions are irregular and variable in distribution: patchy, subpleural and peribronchovascular.

Even if HRCT is more sensitive and specific than chest radiography, it can be normal in cases of histologically proven disease. The differential HRCT diagnosis of subacute allergic extrinsic alveolitis includes DIP, alveolar proteinosis, lymphoid interstitial pneumonia (LIP) and respiratory bronchiolitis-associated interstitial diseases.

\section{Alveolar Proteinosis}

Characterized by the filling of the alveolar spaces with a PAS-positive proteinaceous material rich in lipids, alveolar proteinosis is a rare disease. In most cases, it is idiopathic, even if it is sometimes related to chemotherapeutic treatments, immunologic disorders or malignant neoplasms of the blood.

Radiographic findings in alveolar proteinosis are diffuse alveolar opacities, patchy and bilateral in distribution, with a radiological appearance reminiscent of pulmonary edema. Typical HRCT features of alveolar proteinosis are [41]: ground-glass areas with a geographic distribution associated with a reticular pattern due to septal and intralobular interstitial thickening; the reticular pattern, due to edema or reactive fibrosis, is superimposed on ground-glass attenuation areas (fig. 17). This so-called 'crazy paving' appearance is strictly suggestive, even if not pathognomonic, of alveolar proteinosis.

Other findings are patchy or geographic consolidations, sharply demarcated from the surrounding normal parenchyma, and nodular lesions, with both findings resulting from superimposed infections. Differential diagnosis includes many air space and interstitial lung diseases [69].

The crazy paving appearance for alveolar proteinosis, although being highly suggestive of the disease $(100 \%$ prevalence of this pattern), is not specific.

The main differential diagnosis includes diffuse alveolar damage superimposed on UIP, acute interstitial pneumonia, acute respiratory distress syndrome and bronchoalveolar carcinoma.

Crazy paving can also be found, with lower prevalence, in drug-induced pneumonitis, opportunistic pneumonia, bronchiolitis obliterans with organizing pneumonia, chronic eosinophilic pneumonia, radiation-induced pneumonitis and cardiogenic pulmonary edema [35].

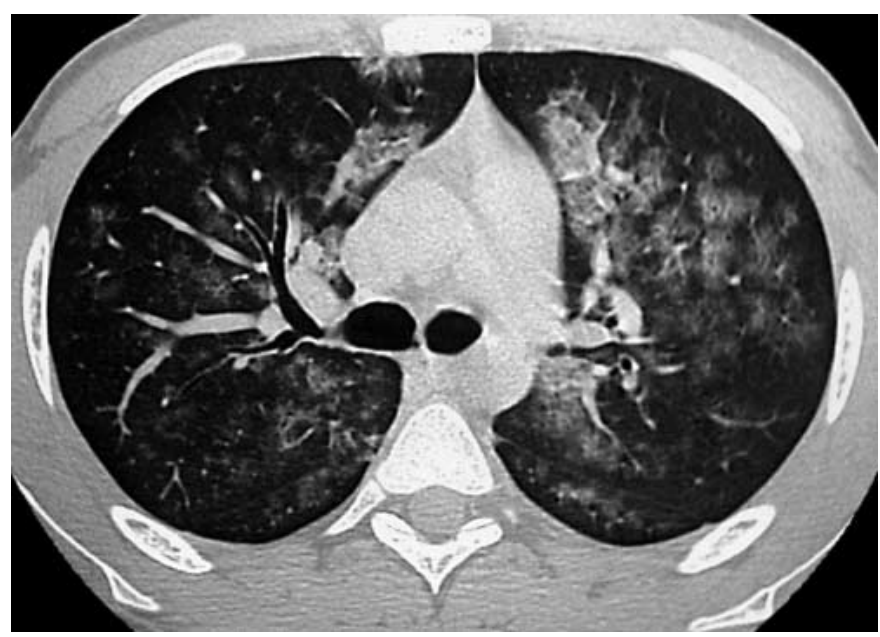

Fig. 17. 48-year-old man with alveolar proteinosis. HRCT scan shows a 'crazy paving' appearance: ground-glass areas with a geographic distribution associated with a superimposed reticular pattern.

\section{Chronic Eosinophilic Pneumonia}

The characteristic peripheral and subpleural consolidations or ground-glass attenuation areas predominant in the upper lobes are better depicted from HRCT than chest $\mathrm{X}$-ray. Chronic eosinophilic pneumonia typically presents the features of a pulmonary edema with an inverted distribution [17, 41, 46]. HRCT can show consolidations with air bronchogram and reactive adenopathy.

Peripheral bands can be found during early regression. In the correct clinical milieu, the features are almost specific for chronic eosinophilic pneumonia. Associated with airspace consolidation, chronic diffuse infiltrative lung diseases include bronchiolitis obliterans with organizing pneumonia (prevalent in the lower lobes), primary lymphoma and bronchoalveolar carcinoma.

\section{Conclusions}

The first diagnostic approach to imaging of diffuse infiltrative disease of the lung remains the chest X-ray. However, because of its limitations in sensitivity, specificity and diagnostic accuracy, a HRCT study is required especially for symptomatic patients with normal or nonspecific chest X-rays.

HRCT is nowadays considered the best imaging tool for diffuse lung diseases, as it plays a crucial investigative role. It is routinely used with excellent results to detect and assess diseases and to predict the response to therapy and likelihood of survival. 
Furthermore, if required, it is used to select the type and location of a biopsy to follow up and assess the response to the medical therapy.

HRCT represents the most important progress in the diagnostic imaging of lung diseases after many years. Compared to chest X-ray, HRCT permits a more detailed and accurate evaluation of the disease, with a more reproducible, comparable and less subjective radiological method: in a word it is more 'scientific'.

New frontiers in lung imaging are to explore with the clinical use of multislice scanners.

Compared with single-slice helical CT, multislice CT can acquire a greater volume (the entire lungs in few seconds) in a single breath hold, with a better longitudinal and in-plane spatial resolution. Multiplanar images of superior quality are available at present; coronal sections enable a diagnostic approach to infiltrative lung diseases, which is as precise as that provided by HRCT scans, with a significant reduction in the number of images [70].

Furthermore, using multislice CT, contiguous chest scans and HRCT sections of superior quality can be obtained by performing a single scan; in this way a comprehensive diagnosis is feasible in patients with suspected focal and diffuse lung disease [71].

In conclusion, today and in the near future, the assessment of diffuse infiltrative lung diseases will be more and more frequently based on a radiological evaluation, starting with the chest X-ray and then increasingly completed with the HRCT.

\section{References}

1 Padley SPG, Hansell DM, Flower CDR, Jennings P: Comparative accuracy of high resolutin computed tomography and chest radiography in the diagnosis of chronic diffuse infiltrative lung disease. Clin Radiol 1991;44:227231.

2 Epler GR, McLoud TC, Gaensler EA, Mikus JP, Carrington CB: Normal chest roentgenograms in chronic diffuse infiltrative lung disease. N Engl J Med 1978;298:934-939.

3 Miller WT Jr: Chest radiographic evaluation of diffuse infiltrative lung disease: Review of a dying art. Eur J Radiol 2002;44:182-197.

4 Mathieson JR, Mayo JR, Staples CA, Müller NL: Chronic diffuse infiltrative lung disease: Comparison of diagnostic accuracy of CT and chest radiography. Radiology 1989;171:111116.

5 Todo G, Itoh H, Nakano Y, Dodo Y, Maeda H, Murata K, Odori T, Torizuka K, Izumi T, Oshima S: High-resolution CT (HR-CT) for the evaluation of pulmonary peripheral disorders. Jpn J Clin Imaging 1982;27:1319-1326.

6 Kazerooni EA: High-resolution CT of the lungs. AJR 2001;177:501-519.

7 Müller NL: Clinical value of high-resolution CT in chronic diffuse lung disease. AJR 1991; 157:1163-1170.

8 Grenier P, Chevret S, Beigelman C, Brauner MW, Chastang C, Valeyre D: Chronic diffuse infiltrative lung disease: Determination of the diagnostic value of clinical data, chest radiography, and CT and Bayesian analysis. Radiology 1994;191:383-390.

9 Heitzman ER: The lung: Radiologic-Pathologic Correlations, ed 3. St. Louis, Mosby, 1984, pp 43-49.

10 Austin JM, Müller NL, Friedman PJ, Hansell DM, Naidich DP, Remy-Jardin M, Webb WR, Zerhouni EA: Glossary of terms for CT of the lungs: Recommendations of the Nomenclature Committee of the Fleischner Society. Radiology 1996;200:327-331.
11 Griffin CB, Primack S: High-resolution CT. Normal Anatomy, Techniques, and Pitfalls. Radiol Clin North Am 2001;39:1073-1090.

12 Murata K, Itoh H, Todo G, Kanaoka M, Noma S, Furuta M, Asamoto H, Torizuka K: Centrilobular lesions of the lung: Demonstration by high-resolution CT and pathologic correlation. Radiology 1986;161:641-645.

13 Webb WR, Stein MG, Finkbeiner WE, Im JG, Lynch D, Gamsu G: Normal and diseased isolated lungs: High-resolution CT. Radiology 1988;166(1 Pt 1):81-87.

14 Hansell DM: High-resolution CT of diffuse lung disease. Value and limitations. Radiol Clin North Am 2001;39:1091-1113.

15 McGuinness G: High Resolution Computed Tomography: Technique and Pitfalls. Seminars in Roentgenology 2002;37:5-16.

16 Webb WR (ed): HRCT of the lung parenchyma. Radiol Clin North Am 1989;27:10851097.

17 Webb WR, Müller NL, Naidich DP: HRCT of the Lung, ed 2. Philadelphia, Lippincott-Raven, 1996.

18 Mayo JR, Webb WR, Gould R, et al: Highresolution CT of the lungs: An optimal approach. Radiology 1987;163:507-510.

19 Webb WR, Müller NL, Naidich DP: HRCT of the Lung, ed 3. Philadelphia, Lippincott, 2001.

20 Murata K, Khan A, Rojas KA, Herman PG: Optimization of computed tomography technique to demonstrate the fine structure of the lung. Invest Radiol 1988;23:170-175.

21 Volpe J, Storto ML, Lee K, Webb WR: Highresolution CT of the lung: Determination of the usefulness of CT scans obtained with the patient prone based on plain radiographic findings. AJR 1997;169:369-374.

22 Arakawa H, Webb WR, McCowin M, Katsou G, Lee KN, Seitz RF: Inhomogeneous lung attenuation at thin-section CT: Diagnostic value of expiratory scans. Radiology 1998;206: 89-94.
23 Webb WR, Stern EJ, Kanth N, Gamsu G Dynamic pulmonary CT: Findings in normal adult men. Radiology 1993;186:117-124.

24 Arakawa H, Webb WR: Air trapping on expiratory high-resolution CT scans in the absence of inspiratory scan abnormalities: Correlation with pulmonary function tests and differential diagnosis. AJR 1998;170:1349-1353.

25 Zwirewich CV, Mayo JR, Müller NL: Lowdose high-resolution CT of lung parenchyma. Radiology 1991;180:413-417.

26 Rotondo A, Guidi G, Catalano O, Smaltino F: High-resolution computerized tomography in the study of the lung parenchyma: Possibility of a low-dose protocol. Radiol Med 1994;87:603607.

27 Majurin ML, Varpula M, Kurki T, Pakkala L: High-resolution CT of the lung in asbestosexposed subjects: Comparison of low-dose and high-dose HRCT. Acta Radiol 1994;35:473477.

28 Majurin ML, Valavaara R, Varpula M, Kurki $\mathrm{T}$, Kulmala J: Low-dose and conventional-dose high-resolution CT of pulmonary changes in breast cancer patients treated by tangential field radiotherapy. Eur J Radiol 1995;20:114119.

29 Gavelli G, Giampalma E, Cenni M, Pierotti L, Cavina M, Bergamini C: High-resolution volumetric computerized tomography of the lung: Optimization of technique and image quality as a function of its clinical-diagnostic use and dose to the patient. Radiol Med 1998;95:322328.

30 Vernhet H, Bousquet C, Vergnes C, Thiebault C, Lesnik A, Durand C, Giron J, Senac JP: Contribution of high-resolution volume computed tomography (HRVCT) for the exploration of diffuse pulmonary infiltrative disorders. Rev Mal Respir 1999;16:188-197.

31 Collins J: CT signs and patterns of lung disease. Radiol Clin North Am 2001;39:1115-1135.

Zompatori/Bnà/Poletti/Spaggiari/Ormitti/ Calabrò/Tognini/Sverzellati 
32 Webb WR, Müller NL, Naidich DP: Standardized terms of high-resolution computed tomography of the lung: A proposed glossary. J Thorac Imaging 1993;8:167-185.

33 McAdams H, Rosato M, Wehunt W, Fishback $\mathrm{N}$ : The alphabet soup revisited. Radiographics 1996;16:1009-1033.

34 Remy-Jardin M, Giraud F, Remy J, Copin MC, Gosselin B, Duhamel A: Importance of ground-glass attenuation in chronic diffuse infiltrative lung disease: Pathologic-CT correlation. Radiology 1993;189:693-698.

35 Schaefer-Prokop C, Prokop M, Fleischamann D, Herold C: High-resolution CT of diffuse interstitial lung disease: Key findings in common disorders. Eur Radiol 2000;11:373-392.

36 Collins J, Stern EJ: Ground-glass opacity at CT: The ABCs. AJR 1997;169:355-367.

37 Lee J, Im JG, Ahn JM, Kim YM, Han MC: Fibrosing alveolitis: Prognostic implication of ground-glass attenuation at high-resolution CT. Radiology 1992;184:451-454.

38 Terriff BA, Kwan SY, Chan-Yeung MM, Müller NL: Fibrosing alveolitis: Chest radiography and CT as predictors of clinical and functional impairment at follow-up in 26 patients. Radiology 1992;184:445-449.

39 Wells AU, Hansell DM, Rubens MB, Cullinan $\mathrm{P}$, Black CM, du Bois RM: The predictive value of appearance on thin section computed tomography in fibrosing alveolitis. Am Rev Respir Dis 1993;148(4 Pt 1):1076-1082.

40 Wells AU, Rubens MB, du Bois RM, Hansel DM: Serial CT in fibrosing alveolitis: Prognostic significance of the initial pattern. AJR 1993; 161:1159-1165.

41 Rotondo A, Grassi R, Catalano O, et al: La patologia interstiziale polmonare nella sclerosi sistemica: caratteristiche semiologiche con la tomografia computerizzata con alta risoluzione e confronto con altre metodiche. Radiol Med 1993;86:213-219.

42 Schurawitzki H, Stiglbauer R, Graininger W, Herold C, Polzeitner D, Burghuber OC, Tscholakoff D: Interstitial lung disease in progressive systemic sclerosis: High-resolution CT versus radiography. Radiology 1990;176:755-759.

43 Heyneman LE, Ward S, Lynch DA, et al: Respiratory bronchiolitis, respiratory bronchiolitis-associated interstitial lung disease, and desquamative interstitial pneumonia: Different entities or part of the spectrum of the same disease process? AJR 1999;173:1617-1622.

44 Yousem S, Colby T, Gaensler E: Respiratory bronchiolitis-associated interstitial lung disease and its relationships to desquamative interstitial pneumonia. Mayo Clin Proc 1989;64: 1373-1380.
45 Lynch D: Imaging of small airways disease. Clin Chest Med 1993;14:623-634.

46 Bonomo L, Ciccotosto C, Gavelli G, Zompatori M: Bronchioliti croniche dell'adulto: TAC ad alta risoluzione del Torace. Gnocchi-Idelson 1996;9:131-150.

47 Aberle DR, Gamsu G, Ray CS: High-resolution CT of benign asbestos-related disease: Clinical and radiographic correlation. AJR 1988; 151:883-891.

48 Leung AN, Staples CA, Müller NL: Chronic diffuse infiltrative lung disease: Comparison of diagnostic accuracy of high-resolution and conventional CT. AJR 1991;157:693-696.

49 Webb WR, Müller NL, Naidich DP: HRCT of the Lung. New York, Raven, 1992, pp 51-87.

50 Goldsmith S, Bailey H, Cattahan E, et al: Pulmonary metastases from breast carcinoma. Arch Surg 1967;94:483-488.

51 Noma S, Khan A, Herman PG, et al: Highresolution computed tomography of the pulmonary parenchyma. Semin Ultrasound CT MR 1990;11:365-379.

52 Romagnoli R, Bertolani M, Guicciardi L: Diagnostica per immagini della patologia interstiziale non infettiva. Rays 1992;17:242-261.

53 Spencer H: Pathology of the Lung. Oxford, Pergamon Press, 1985, p 1085.

54 Stein M, Mayo J, Müller NL, et al: Pulmonary lymphangitic spread of carcinoma: Appearance on CT scan. Radiology 1987;162:371-375.

55 Grenier P, Valeyre D, Cluzel P, Brauner MW, Lenoir S, Chastang C: Chronic diffuse interstitial lung disease: Diagnostic value of chest radiography and high-resolution CT. Radiology 1991;179:123-132.

56 Crystal RG, Sarcoidosis; in Fauci AS, Braunwald $\mathrm{E}$, Isselbacher $\mathrm{KJ}$, Wilson JD, Martin JB, Kasper DL, Hanser SL, Longo DL (eds): Harrison: Principi di Medicina Interna, ed 14. Trento, McGraw-Hill, 1999, pp 2192-2198.

57 Nishimura K, Itoh H, Kitaichi M, Nagai S, Izumi T: Pulmonary sarcoidosis: Correlation of CT and histopathologic findings. Radiology 1993; 189:105-109.

58 Bergin CJ, Bell DJ, Coblentzl CL, Chiles C, Gamsu G, MacIntyre NR, Coleman RE, Putman CE: Sarcoidosis: Correlation of pulmonary parenchymal pattern at CT with results of pulmonary function tests. Radiology 1989;171: 619-624

59 Müller NL, Kullnig P, Miller RR: The CT findings of pulmonary sarcoidosis: Analysis of 25 patients. AJR 1989;152:1179-1182.

60 Brauner MW, Lenoir S, Grenier P, Clutzel P, Battesti JP, Valeyre D: Pulmonary sarcoidosis: CT assessment of lesion reversibility. Radiology 1992;182:349-354.
61 Nakata H, Kimoto T, Nakayama T, Kido M, Miyazaki N, Harada S: Diffuse peripheral lung disease: Evaluation by high-resolution computed tomography. Radiology 1985;157:181185.

62 Lynch DA, Webb WR, Gamsu G, et al: Computed tomography in pulmonary sarcoidosis. $\mathrm{J}$ Comput Assist Tomogr 1989;13:405-410.

63 Austin JM: Pulmonary sarcoidosis: What are we learning from CT? Radiology 1989;171: 603-605.

64 Müller NL, Mawson JB, Mathieson JR, Abboud R, Ostrow DN, Champion P: Sarcoidosis: Correlation of extent of disease with clinical, functional and radiographic findings. Radiology 1989;171:613-618.

65 Remy-Jardin M, Giraud F, Remy J, Wattinne L, Wallaert B, Duhamel A: Pulmonary sarcoidosis: Role of CT in the evaluation of disease activity and functional impairment and prognosis assessment. Radiology 1994;191:675680.

66 Hansell DM, Milne DG, Wilsher ML, Wells AU: Pulmonary sarcoidosis: Morphologic associations of airflow obstruction at thin section CT. Radiology 1998;209:697-704.

67 Speizer FE: Patologie polmonari da agenti ambientali; in Fauci AS, Braunwald E, Isselbacher KJ, Wilson JD, Martin JB, Kasper DL, Hanser SL, Longo DL (eds): Harrison: Principi di Medicina Interna, ed 14. McGraw-Hill, Trento, 1999, pp 1638-1639.

68 Hunninghake GW, Richerson HB: Polmoniti da ipersensibilità e polmoniti eosinofile; in Fauci AS, Braunwald E, Isselbacher KJ, Wilson JD, Martin JB, Kasper DL, Hanser SL, Longo DL (eds): Harrison: Principi di Medicina Interna, ed 14. McGraw-Hill, Trento, 1999, pp 1632-1636.

69 Johkoh T, Itoh H, Müller NL, Ichikado K, Nakamura H, Ikezoe J, Akira M, Nagareda T: Crazy-paving appearence at thin section $\mathrm{CT}$ : Spectrum of disease and pathologic finding. Radiology 1999;211:155-160.

70 Remy-Jardin M, Campistron P, Amara A, Mastora I, Tillie-Leblond I, Delannoy V, Duhamel A, Remy J: Usefulness of coronal reformations in the diagnostic evaluation of infiltrative lung disease. J Comput Assist Tomogr 2003;27:266-273.

71 Schoepf UJ, Bruening RD, Hong C, Eibel R, Aydemir S, Crispin A, Becker C, Reiser M: Multislice helical CT of focal and diffuse lung disease: Comprehensive diagnosis with reconstruction of contiguous and high-resolution CT sections from a single thin-collimation scan. AJR 2001;177:179-184. 